\title{
The Numerical Evaluation of Double Integrals.
}

\author{
By A. C. Aitken and G. L. Frewin.
}

(Read 7th December 1928. Received 25th December 1923.)

\section{\$1. Introduction.}

The present paper is concerned with formulae by which double integrals of functions of two independent variables mas be evaluated approximately. The number of such formulae published hitherto is not great, ${ }^{*}$ and it has seemed desirable both to make a systematic search for new formulae, and to test the comparative merits of these, and of those previously known, by computing the numerical values of certain selected integrals.

\section{\$2. General Formulae.}

Take a known formula for the numerical evaluation of single integrals, say

$$
\frac{1}{u} \int_{a}^{a+m u} f(x) d x=\sum_{r} A_{r} f(a+r u)+\sum_{r} A_{r}^{\prime} \phi_{r}\left(\Delta_{x}\right) f(a+r u),
$$

and similarly

$$
\frac{1}{v} \int_{b}^{b+n v} f(y) d y=\sum_{s} B_{\imath} f(b+s v)+\sum_{s} B_{s}^{\prime} \psi_{s}\left(\Delta_{y}\right) f(b+s v),
$$

where the $A_{r}, A_{r}^{\prime}, B_{\imath}, B_{1}^{\prime}$ are given coefficients, $\phi_{r}$ and $\psi_{\text {f functions }}$ of the symbols of partial finite difference operating on the functions to be integrated.

Then a general formula for the numerical evaluation of double integrals is

$$
\begin{array}{r}
\frac{1}{u v} \int_{a}^{a+m u} \int_{b}^{b+n v} f(x, y) d x d y=\frac{1}{u} \int_{a}^{a+m u}\left\{\Sigma B_{a} f(x, b+s v)\right. \\
\left.+\Sigma B_{s}^{\prime} \psi_{s}\left(\Delta_{y}\right) f(x, b+s v)\right\} d x \\
=\Sigma \Sigma A_{r} B_{s} f(a+r u, b+s v)+\Sigma \Sigma A_{r}^{\prime} B_{s} \phi_{r}\left(\Delta_{x}\right) f(a+r u, b+s v) \\
+\Sigma \Sigma A_{r} B_{s}^{\prime} \psi_{s}\left(\Delta_{y}\right) f(a+r u, b+8 v) \\
+\Sigma \Sigma A_{r}^{\prime} B_{s}^{\prime} \phi_{r}\left(\Delta_{x}\right) \psi_{s}\left(\Delta_{y}\right) f(a+r u, b+s v),
\end{array}
$$

a symbolic product of the single formulae from which it is derived.

- The matter is discussed briefly in $\$ 182$ of Whittaker and Robinson's "Calculus of Observations," where references are given. 
Again, if differential coefticients are involved, instead of differences, say

$$
\begin{aligned}
& \frac{1}{u} \int_{a}^{a+m u} f(x) d x=\sum A_{r} f(a+r u)+\sum A_{r}^{\prime} \phi_{r}\left(u \frac{\hat{o}}{\partial x}\right) f(a+r u), \\
& \frac{1}{v} \int_{b}^{b+n v} f(y) d y=\Sigma B_{s} f(b+s v)+\sum B_{s}^{\prime} \psi_{s}\left(v \frac{\partial}{\partial y}\right) f(b+s v),
\end{aligned}
$$

it follows in similar fashion that

$$
\begin{aligned}
\frac{1}{u v} \int_{a}^{a+m u} \int_{b}^{b+n v} f( & x, y) d x d y=\Sigma \Sigma A_{r} B_{s} f(a+r u, b+s v) \\
& +\sum \Sigma A_{r}^{\prime} B_{s} \phi_{r}\left(u \frac{\partial}{\partial x}\right) f(a+r u, b+s v) \\
& +\sum \Sigma A_{r} B_{s}^{\prime} \psi_{s}\left(v \frac{\partial}{\partial y}\right) f(a+r u, b+s v) \\
& +\Sigma \Sigma A_{r} B_{s}^{\prime} \phi_{r}\left(u \frac{\partial}{\partial x}\right) \psi_{*}\left(v \frac{\partial}{\partial y}\right) f(a+r u, b+s v) .
\end{aligned}
$$

In many cases we have

$$
\frac{1}{u} \int_{a}^{a+m u} f(x) d x=\mathrm{\Sigma} A_{r} f(a+r u)+R_{x}
$$

where $R_{x}$ is a remainder of the form $A_{h}^{\prime}\left(\frac{\partial}{\partial x}\right)^{p} f(a+h u)$, $a+h u$ being some value of $x$ within the range, and likewise

Then

$$
\frac{1}{v} \int_{b}^{b+n v} f(y) d y=\Sigma B_{s} f(b+s v)+B_{k}^{\prime}\left(\frac{\partial}{\partial y}\right)^{t} f(b+k v) .
$$

$$
\begin{aligned}
& \frac{1}{u v} \int_{a}^{a+m u} \int_{b}^{b+n v} f(x, y) d x d y=\Sigma \Sigma A_{r} B_{s} f(a+r u, b+s v) \\
& \quad+\Sigma A_{h}^{\prime} B_{a}\left(\frac{\partial}{\partial x}\right)^{p} f(a+h u, b+s v) \\
& \quad+\Sigma A_{r} B_{k}^{\prime}\left(\frac{\partial}{\partial y}\right)^{q} f(a+r u, b+k v) \\
& \quad+A_{h}^{\prime} B_{k}^{\prime}\left(\frac{\partial}{\partial x}\right)^{p}\left(\frac{\partial}{\partial y}\right)^{q} f(a+h u, b+k v) .
\end{aligned}
$$

The last three terms of this expression form the remainder $R_{x_{1} y}$, but in practice the last term is negligible. 


\section{§3. Particular Formulae: Extended Newton-Cotes Formulae.}

The simplest and generally the least accurate of the common approximations to a single integral is the "trapezoidal" rule, viz., $\frac{1}{u} \int_{a}^{a+m u} f(x) d x=\frac{1}{2} f(a)+\sum_{r=1}^{m-1} f(a+r u)+\frac{1}{2} f(a+m u)$ nearly.

For a double integral, by $\S 2$, the corresponding formula is $\frac{1}{u v} \int_{a}^{a+m u} \int_{b}^{b+n v} f(x, y) d x d y=\frac{1}{4} f(a, b)+\frac{1}{2} \sum_{s=1}^{n-1} f(a, b+s v)$ $+\frac{1}{4} f(a, b+n v)+\frac{1}{2} \sum_{r=1}^{m-1} f(a+r u, b)+\sum_{r=1}^{m-1} \sum_{s=1}^{n-1} f(a+r u, b+8 v)$ $+\frac{1}{2} \sum_{r=1}^{m-1} f(a+r u, b+n v)+\frac{1}{4} f(a+m u, b)+\frac{1}{2} \sum_{s=1}^{n-1} f(a+m u, b+s v) \cdot$ $+\frac{1}{4} f(a+m u, b+n v)$ nearly.

In fact, the coefficients for one variable being given in a row as $\frac{1}{2}, 1,1, \ldots 1, \frac{1}{2}$

those for two variables may be tabulated in rows and columns as

$$
\mid \begin{aligned}
& \frac{1}{4}, \frac{1}{2}, \frac{1}{2}, \ldots \frac{1}{2}, \frac{1}{4}, \\
& \frac{1}{2}, 1,1, \ldots 1, \frac{1}{2}, \\
& \ldots \ldots \ldots \ldots \ldots \ldots . . . \\
& \ldots \ldots \ldots \ldots \ldots \ldots . . . \\
& \frac{1}{4}, \frac{1}{2}, \frac{1}{2}, \ldots \frac{1}{2}, \frac{1}{4} .
\end{aligned}
$$

We shall find it convenient to refer to the sums of values of a function taken with these coefticients as "trapezoidal" sums, denoting by $S_{u}^{m} f(a)$ the $\operatorname{sum} \frac{1}{2} f(a)+\sum_{r=1}^{m-1} f(a+r u)+\frac{1}{2} f(a+m u)$, and similarly for $S_{u}^{m} S_{v}^{n} f(a, b)$.

The extension to a triple integral is easily seen. The coefficients of $S_{u}^{m} S_{v}^{n} S_{w}^{p} f(a, b, c)$ can be represented tier upon tier, forming a cube, the coefficients at the corners being $\frac{1}{8}$, along the edges $\frac{1}{4}$, in the faces $\frac{1}{2}$, and within the cube 1 .

The double "Simpson" formula is

$$
\begin{aligned}
\frac{1}{u v} \int_{a}^{a+2 u} \int_{b}^{b+2 v} f(x, y) d x d y=\frac{1}{9}\{f(a, b)+4 f(a+u, b) \\
\quad+f(a+2 u, b)+4 f(a, b+v)+16 f(a+u, b+v) \\
\quad+4 f(a+2 u, b+v)+f(a, b+2 v)+4 f(a+u, b+2 v) \\
\quad+f(a+2 u, b+2 v)\} \text { nearly. }
\end{aligned}
$$


Accuracy may generally be increased by having more subdivisions, when coefficients are obtained as below :-

$$
\frac{u v}{9} \times \quad \begin{array}{rrrrrrrr}
1, & 4, & 2, & 4, & 2, & \ldots & 4, & 1 \\
4, & 16, & 8, & 16, & 8, & \ldots & 16, & 4 \\
2, & 8, & 4, & 8, & 4, & \ldots & 8, & 2 \\
4, & 16, & 8, & 16, & 8, & \ldots & 16, & 4 \\
2, & 8, & 4, & 8, & 4, & \ldots & 8, & 2 \\
\ldots & \ldots & \ldots & \ldots & \ldots \ldots & \ldots \ldots \\
4, & 16, & 8, & 16, & 8, & \ldots & 16, & 4 \\
1, & 4, & 2, & 4, & 2, & \ldots & 4, & 1
\end{array}
$$

Example.-The true value of $\int_{0}^{1} \int_{0}^{1}\left(1+x^{2}+y^{2}\right)^{-\frac{\pi}{2}} d x d y$ is $\frac{\pi}{6}$, or .523599 to six places.

A first crude application of the elementary Simpson formula for nine points gave the result $\cdot 5195$.

For 25 points, $\cdot 52363$ was obtained. 49 points gave the much closer result $\cdot 523609$, and 121 points 52.3602 .

Corresponding to the "Three-Eighths" or Simpson's Second Rule there is the scheme

$$
\frac{9 u v}{64} \times \quad \begin{array}{ccccccccc}
1, & 3, & 3, & 2, & 3, & 3, & \ldots & 3, & 1 \\
3, & 9, & 9, & 6, & 9, & 9, & \ldots & 9, & 3 \\
3, & 9, & 9, & 6, & 9, & 9, & \ldots & 9, & 3 \\
2, & 6, & 6, & 4, & 6, & 6, & \ldots & 6, & 2 \\
3, & 9, & 9, & 6, & 9, & 9, & \ldots & 9, & 3 \\
\ldots & \ldots \ldots \ldots \ldots \ldots \ldots \ldots & \ldots \ldots \ldots & \ldots & \ldots & \ldots & \ldots & \ldots & \ldots \\
3, & 9, & 9, & 6, & 9, & 9, & \ldots & 9, & 3 \\
1, & 3, & 3, & 2, & 3, & 3, & \ldots & 3, & 1 .
\end{array}
$$

49 values of the same function as above gave the result by this formula 523591 .

It is possible and sometimes convenient to combine the Simpson with the Three-Eighths Rule, obtaining the scheme

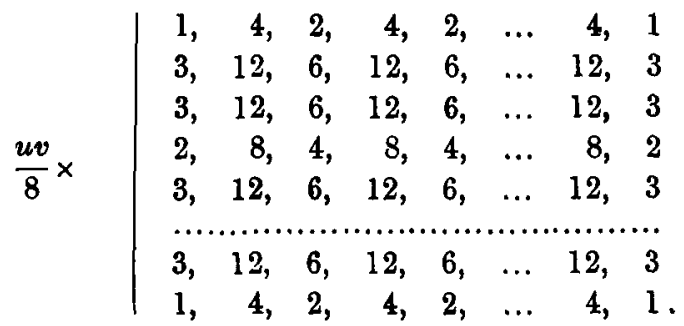


$\int_{0}^{\cdot 8} \int_{0}^{1 \cdot 2} e^{x^{x} y} d x d y$ was calculated by this formula for 117 values, the result $1 \cdot 10467$ being obtained. The true value is $1 \cdot 10469$.

A fairly accurate formula when the ranges may conveniently be subdivided into six intervals or some multiple of six, is the double Weddle formula, viz. :

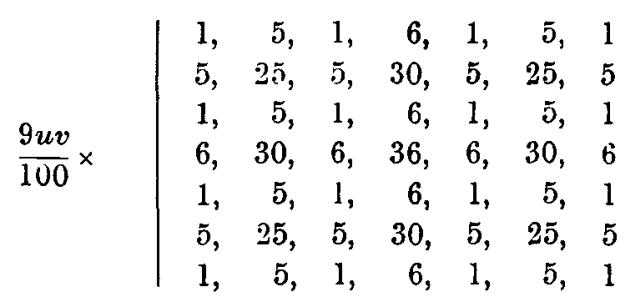

This formula gave for $\int_{0}^{1} \int_{0}^{1}\left(1+x^{2}+y^{2}\right)^{-\frac{3}{2}} d x d y$ the result .523602 , to a degree of accuracy for 49 points equal to that of the Simpson formula for 121 points.

A great number of other formulae may be obtained by combining single formulae in pairs, but it is not necessary to exemplify these.

It is of course possible to obtain analogous formulae for multiple integrals. Thus for a triple integral such as

$$
\int_{a}^{a+2 m u} \int_{b}^{b+2 n v} \int_{c}^{c+? p v o} f(x, y, z) d x d y d z
$$

Simpson coefficients may be given in three tiers of nine, one above another :

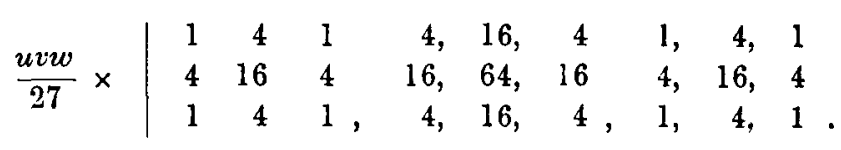

It may be remarked that the accurate evaluation of a double or of a multiple integral requires in most cases the addition of a large number of terms. It is here that calculating machines, and adding machines in particular, are of the greatest service and almost indispensable. 
\$ 4. Extended Gauss Formulas.

Combination of Gauss formulae of the types

$\int_{-1}^{1} f(x) d x=f\left(\sqrt{\frac{1}{3}}\right)+f\left(-\sqrt{\frac{1}{3}}\right)$

$$
=\frac{1}{9}\left\{5 f\left(\sqrt{\frac{5}{5}}\right)+8 f(0)+5 f\left(-\sqrt{\frac{3}{5}}\right)\right\}
$$

gives formulae such as

$$
\begin{aligned}
& \int_{-1}^{1} \int_{-1}^{1} f(x, y) d x d y=f\left(\sqrt{\frac{1}{3}},\right.\left.\sqrt{\frac{1}{3}}\right)+f\left(\sqrt{\frac{1}{3}}, \cdots \sqrt{\frac{1}{3}}\right) \\
&+f\left(-\sqrt{\frac{1}{3}}, \sqrt{\frac{1}{3}}\right)+f\left(-\sqrt{\frac{1}{3},}-\sqrt{\frac{1}{3}}\right) \\
&=\frac{1}{9}\left[8\left\{f\left(0, \sqrt{\frac{1}{3}}\right)+f\left(0,-\sqrt{\frac{1}{3}}\right)\right\}\right.+5\left\{f\left(\sqrt{\frac{3}{5}}, \sqrt{\frac{1}{3}}\right)\right. \\
&\left.+f\left(\sqrt{\frac{3}{5}},-\sqrt{\frac{1}{3}}\right)+f\left(-\sqrt{\frac{3}{5}}, \sqrt{\left.\frac{1}{3}\right)}+f\left(-\sqrt{\frac{3}{5}},-\sqrt{\frac{1}{3}}\right)\right\}\right] \\
&=\frac{1}{81}\left[64 f(0,0)+40\left\{f\left(0, \sqrt{\frac{3}{5}}\right)+f\left(0, \cdots \sqrt{\frac{3}{5}}\right)+f\left(\sqrt{\frac{3}{5}}, 0\right)\right.\right. \\
&\left.+f\left(-\sqrt{\frac{3}{5}}, 0\right)\right\}+25\left\{f\left(\sqrt{\frac{3}{5}}-\sqrt{\frac{3}{5}}\right)\right. \\
&\left.\left.+f\left(\sqrt{\frac{3}{3}},-\sqrt{\frac{3}{5}}\right)+f\left(-\sqrt{\frac{3}{5}}, \sqrt{\frac{3}{5}}\right)+f\left(-\sqrt{\frac{3}{5}},-\sqrt{\frac{3}{5}}\right)\right\}\right] .
\end{aligned}
$$

The last of these is exact if neither of $f(x, 1)$ and $f(1, y)$ is of higher than the fifth degree.

For $\int_{0}^{1} \int_{0}^{1}\left(1+x^{2}+y^{2}\right)^{-\hat{s}} d x d y$ this formula gives the result .5233. For $\int_{0}^{1} \int_{0}^{1} e^{x^{2} y} d x d y$ it gives 1.20698 , the true value to five places being 1.20702. A Simpson of 121 points gave 1.20703.

We may mention here a formula of Burnside derived by a different method,

$$
\begin{aligned}
& \int_{-1}^{1} \int_{-1}^{1} f(x, y) d x d y=\frac{40}{4}\left\{f\left(\sqrt{\frac{7}{15}}, 0\right)\right.+f\left(-\sqrt{\frac{7}{15}}, 0\right) \\
&\left.+f\left(0, \sqrt{\frac{7}{15}}\right)+f\left(0,-\sqrt{\frac{7}{15}}\right)\right\} \\
&+\frac{9 g}{49}\left\{f\left(\sqrt{\frac{7}{9}}, \sqrt{\frac{7}{9}}\right)+f\left(\sqrt{\frac{7}{9}},-\sqrt{\frac{7}{9}}\right)+f\left(-\sqrt{\frac{7}{9}}, \sqrt{\frac{7}{9}}\right)\right. \\
&\left.+f\left(-\sqrt{\frac{7}{9}},-\sqrt{\frac{7}{9}}\right)\right\} .
\end{aligned}
$$

For $\int_{0}^{1} \int_{0}^{1}\left(1+x^{2}+y^{3}\right)^{-1} d x d y$ this formula gives 5232 . For $\int_{0}^{1} \int_{0}^{1}\left(3-x^{2}-y^{2}\right)^{-\frac{1}{2}} d x d y$ it gives 6641 , and for $\int_{0}^{1} \int_{0}^{1}\left(2-x^{2}-y^{2}\right)^{-\frac{1}{2}} d x d y$ .9262 , the true values being $\cdot 6638$ and .9202 . Results obtained by the third Gauss formula given above are slightly better, being .6638 and 9144 . 
The amount of computation required by the two formulae is about the same.

All formulae such as the Simpson, Weddle, etc., involve the use of corner points of the range of integration, and therefore fail in such a case as $\int_{0}^{1} \int_{0}^{1}\left(2-x^{2}-y^{2}\right)^{-\frac{1}{3}} d x d y$, where the integrand becomes infinite at the point $x=1, y=1$. To evaluate such integrals we may combine a formula involving end points with a formula not involving end points. Thus a combination of elementary Gauss and Simpson formulae gives

$$
\begin{array}{r}
\int_{-1}^{1} \int_{-1}^{1} f(x, y) d x d y=\frac{1}{3}\left[f\left(\sqrt{\frac{1}{3}}, 1\right)+f\left(\sqrt{\frac{1}{3}},-1\right)+f\left(-\sqrt{\frac{1}{3}}, 1\right)\right. \\
\left.+f\left(-\sqrt{\frac{1}{3}},-1\right)+4\left\{f\left(\sqrt{\frac{1}{3}}, 0\right)+f\left(-\sqrt{\frac{1}{3}}, 0\right)\right\}\right] .
\end{array}
$$

This gives for $\int_{0}^{1} \int_{0}^{1}\left(2-x^{2}-y^{2}\right)^{-1} d x d y$ the value $\cdot 9205$, but the accuracy of the result is possibly accidental.

\section{\$5. Chebyshef Formulae.}

Combination of Chebyshef formulae of the types

$$
\begin{aligned}
\int_{-1}^{1} f(x) d x & =\frac{2}{3}\left\{f(-p)+f(0)+f^{\prime}(p)\right\} \\
& =\frac{2}{5}\{f(-q)+f(-r)+f(0)+f(r)+f(q)\},
\end{aligned}
$$

where $p=707166 \ldots, q=832437 \ldots, \quad r=.374542 \ldots, \quad$ gives formulae such as

$$
\begin{aligned}
\int_{-1}^{1} \int_{-1}^{1} f^{\prime}(x, y) & d x d y=\frac{4}{9}\left\{f^{\prime}(p, p)+f(p,-p)+f(-p, p)\right. \\
& +f(-p,-p)+f(0,0)+f(p, 0)+f(-p, 0) \\
& +f(0, p)+f(0,-p)\},
\end{aligned}
$$

and other formulae of a greater number of terms.

Such formulae have the advantage that the terms may be summed without first multiplying by various coefficients, but even so the values of the variables required in them are not very convenient for ordinary applications. The simplest of these formulae, 
written out above, gives fair results, e.g. for $\int_{0}^{1} \int_{0}^{1}\left(1+x^{2}+y^{2}\right)^{-\frac{3}{2}} d x d y$ $\int_{0}^{1} \int_{0}^{1}\left(2-x^{2}-y^{2}\right)^{-\frac{1}{2}} d x d y$ and $\int_{0}^{1} \int_{0}^{1}\left(3-x^{2}-y^{2}\right)^{-\frac{1}{2}} d x d y$ the values $\cdot 5245, \cdot 9107$ and $\cdot 6645$ respectively.

\section{§6. Formulae in Finite Differences.}

These formulae are of use where the values of the integrand are tabulated for equidistant values of the variables. As in the case of a function of one variable, they consist of terms giving a trapezoidal sum, followed by correction terms, but while in the former case these terms refer to the ends of the linear range of integration, here they refer to the boundary of the rectangular region.

Corresponding to the single formula

$$
\begin{aligned}
& \frac{1}{u} \int_{a}^{a+m u} f(x) d x=S_{u}^{m} f(a)-\frac{\Delta}{12}\{f(a+m u)-f(a)\} \\
&+\frac{\Delta^{2}}{24}\{f(a+m u)-f(a)\} \\
&-\frac{19 \Delta^{3}}{720}\{f(a+m u)-f(a)\}+\frac{3 \Delta^{4}}{160}\{f(a+m u)-f(a)\}-\text { etc., }
\end{aligned}
$$

we have by $\S 2$ the double formula

$$
\begin{aligned}
& \frac{1}{u v} \int_{a}^{a+m u} \int_{b}^{b+n v} f(x, y) d i u d y=S_{u}^{m} S_{v}^{n} f(a, b) \\
&-\frac{\Delta x}{12}\left[S_{u}^{0} S_{v}^{n}\{f(a+m u, b)-f(a, b)\}\right] \\
&-\frac{\Delta y}{12}\left[S_{u}^{m} S_{v}^{0}\{f(a, b+n v)-f(a, b)\}\right] \\
&+\frac{\Delta_{x}^{2}}{24}\left[S_{u}^{0} S_{v}^{n}\{f(a+m u, b)-f(a, b)\}\right] \\
&+\frac{\Delta_{y}^{2}}{24}\left[S_{u}^{m} S_{v}^{0}\{f(a, b+n v)-f(a, b)\}\right] \\
&-\frac{19 \Delta_{x}^{3}}{720}[\text { etc. ... }]-\ldots+\frac{\Delta_{x} \Delta_{y}}{144}[f(a+m u, b+n v)-f(a, b+n v)-f(a+m u, b)+f(a, b)] \\
&-\frac{\Delta_{x}^{2} \Delta_{y}+\Delta_{x} \Delta_{v}^{2}}{288}[f(a+m u, b+n v)-\text { etc. }]+\ldots,
\end{aligned}
$$

where $S_{u}^{0} f(a)=f^{\prime}(a)$. 
The mode of applying the formula can best be illustrated from the actual table below of $z=e^{x y}$.

\begin{tabular}{|c|c|c|c|c|c|}
\hline & & & & & \\
\hline & $\cdot 4$ & .5 & 6 & .7 & $\cdot 8$ \\
\hline $1 \cdot 3$ & $1 \cdot 2312$ & $1 \cdot 3840$ & $1 \cdot 5968$ & $1 \cdot 8908$ & $2 \cdot 2979$ \\
\hline $1 \cdot 4$ & $1 \cdot 2511$ & $1 \cdot 4191$ & $1 \cdot 6553$ & 1.9858 & $2 \cdot 4498$ \\
\hline 1.5 & $1 \cdot 2712$ & $1 \cdot 4550$ & 1.7160 & $2 \cdot 0855$ & $2 \cdot 6117$ \\
\hline $\begin{array}{ll}y & 1 \cdot 6\end{array}$ & $1 \cdot 2918$ & 1.4918 & $1 \cdot 7789$ & $2 \cdot 1902$ & $2 \cdot 7843$ \\
\hline $1 \cdot 7$ & $1 \cdot 3126$ & 1.5296 & $1 \cdot 8441$ & $2 \cdot 3002$ & $2 \cdot 9683$ \\
\hline $1 \cdot 8$ & $1 \cdot 3338$ & $1 \cdot \overline{5683}$ & 1.9117 & $2 \cdot 4157$ & $3 \cdot 1645$ \\
\hline
\end{tabular}

The terms $S_{u}^{0} S_{v}^{u} f(a, b)$ and similar ones mean the trapezoidal sums of columns, $S_{u}^{m} S_{v}^{0} f(a, b)$ the trapezoidal sums of rows.

Hence the best method is first to take trapezoidal sums of columns and rows, and construct two difference tables of these. (For the above formula columns and rows outside the range are necessary in order to obtain the required differences.)

The trapezoidal sum of either these sums of columns or the sums of rows gives the trapezoidal approximation to the integral, $S_{u}^{m} S_{v}^{n} f(a, b)$. The more important correction terms are obtained from the two difference tables, just as for single numerical integration. As for the terms in $\Delta_{x} \Delta_{y}, \Delta_{x}^{2} \Delta_{y}, \Delta_{x} \Delta_{y}^{2}$, etc, they are small corrections taken at the four corners, and are generally negligible.

When values of the integrand within the range of integration only are available, e.g. if from the table above we have to find

$$
\begin{aligned}
& \int_{-4}^{.8} \int_{1 \cdot 3}^{r-8} e^{x z y} d x d y, \text { we may use the similar formula } \\
& \begin{aligned}
\frac{1}{u v} \int_{a}^{a+m u} \int_{b}^{b+n v} f(x, y) d x d y & =S_{u}^{m} S_{v}^{n} f(a, b) \\
& -\frac{\Delta_{x}}{12}\left[S_{u}^{0} S_{v}^{n}\{f(a+\overline{m-1} u, b)-f(a, b)\}\right] \\
& -\frac{\Delta_{y}}{12}\left[S_{u}^{m} S_{v}^{0}\{f(a, b+\overline{n-1} v)-f(a, b)\}\right] \\
& -\frac{\Delta_{x}^{2}}{24}\left[S_{u}^{0} S_{v}^{n}\{f(a+\overline{m-2} u, b)+f(a, b)\}\right]
\end{aligned}
\end{aligned}
$$




$$
\begin{array}{r}
\left.-\frac{\Delta_{y}^{2}}{24} S_{u}^{n} S_{v}^{0}\{f(a, b+\overline{n-2} v)+f(a, b)\}\right] \\
-\frac{19 \Delta_{x}^{s}}{720}\left[S_{u}^{o} S_{v}^{n}\{f(a+\overline{m-3} u, b)-f(a, b)\}\right] \\
-\frac{19 \Delta_{v}^{3}}{720}[\text { etc. } \ldots]+\ldots+\frac{\Delta_{x} \Delta_{y}}{144}[f(a+\overline{m-1} u, b+\overline{n-1} v) \\
-f(a, b+\overline{n-1} v)-f(a+\overline{m-1} u, b)+f(a, b)]+\ldots
\end{array}
$$

Example. -From the given table of values of $e^{x^{3} y}$ the trapezoidal sums of columns are $6.4092,7 \cdot 3716,8 \cdot 7485,10 \cdot 7149,13.5453$, of rows $6 \cdot 6361,6 \cdot 9106,7 \cdot 1979,7 \cdot 4989,7 \cdot 8143,8 \cdot 1448$.

The trapezoidal sum of either of these sets, with the differences of successive orders, gives approximations to $\int_{-4}^{-8} \int_{1 \cdot 3}^{1 \cdot 8} e^{x^{2} y} d x d y$ of $\cdot 36812, \cdot 36652, \cdot 36598, \cdot 36595, \cdot 36591$, the last being correct to five places. Corner corrections were neglected.

As a further test, $z=\left(1+x^{2}+y^{2}\right)^{-3}$ was tabulated at intervals of 1 between the values $x=0,1, y=0,1$. To the third order of differences this gives for $\int_{0}^{1} \int_{0}^{1}\left(1+x^{2}+y^{2}\right)^{-1} d x d y$ the good approximation $\cdot 523604$.

If values of $x$ outside its range of integration are available, but not of $y$ outside its range, a compromise between the two preceding formulae may be given :

$$
\begin{aligned}
& \frac{1}{u v} \int_{a}^{a+m u} \int_{b}^{b+n v} f(x, y) d x d y=S_{u}^{m} S_{v}^{n} f(a, b) \\
&-\frac{\Delta_{x}}{12}\left[S_{u}^{0} S_{v}^{n}\{f(a+m u, b)-f(a, b)\}\right] \\
&-\frac{\Delta_{v}}{12}\left[S_{u}^{m} S_{v}^{0}\{f(a, b+\overline{n-1} v)-f(a, b)\}\right] \\
&+\frac{\Delta_{x}^{2}}{24}\left[S_{u} S_{v}^{n}\{f(a+m u, b)-f(a, b)\}\right] \\
&-\frac{\Delta_{v}^{2}}{24}\left[S_{u}^{m} S_{v}^{0}\{f(a, b+\overline{n-2} v)+f(a, b)\}\right]-\frac{19 \Delta_{x}^{3}}{720}[\text { etc. }] \\
&-\frac{19 \Delta_{v}^{3}}{720}[\text { etc. }]+\ldots+\frac{\Delta_{x} \Delta_{y}}{144}\left[f^{\prime}(a+m u, b+\overline{n-1 v})-f(a, b+\overline{n-1} v)\right. \\
&-f(a+m u, b)+f(a, b)]+\ldots \text { etc. }
\end{aligned}
$$


From the Bessel central difference formula

$$
\begin{aligned}
\frac{1}{u} \int_{a}^{a+m u} f(x) d x=S_{u}^{m} f(a)-\frac{\mu \delta}{12}\left(f_{m}-f_{0}\right)+\frac{11 \mu \delta^{3}}{720} & \left(f_{m}-f_{0}\right) \\
& -\frac{191 \mu \delta^{8}}{60480}\left(f_{m}-f_{0}\right)+\ldots,
\end{aligned}
$$

where $\mu \delta f_{r}^{\prime}=\frac{1}{2}\left(\delta f_{r+t}+\delta f_{r-\frac{1}{\xi}}\right)$ in the usual notation, we derive by $\S 2$

$$
\begin{aligned}
& \frac{1}{u v} \int_{a}^{a+m u} \int_{b}^{b+n v} f(x, y) d x d y=S_{u}^{m} S_{v}^{n} f(a, b)-\frac{\mu_{x} \delta_{x}}{12}\left[S_{u}^{0} S_{v}^{n}\left(f_{m, 0}-f_{0,0}\right)\right]-\frac{\mu_{y} \delta_{y}}{12}\left[S_{u}^{m} S_{v}^{0}\left(f_{0, n}-f_{0,0}\right)\right] \\
&+\frac{11 \mu_{x} \delta_{x}^{3}}{720}\left[S_{u}^{0} S_{v}^{n}\left(f_{m, 0}-f_{0,0}\right)\right]+\frac{11 \mu_{\nu} \delta_{v}^{3}}{720}\left[S_{u}^{m} S_{v}^{0}\left(f_{0, n}-f_{0,0}\right)\right]-\ldots \\
&+\frac{\mu_{x} \delta_{x} \mu_{y} \delta_{y}}{144}\left(f_{m, n}-f_{0, n}-f_{m, 0}+f_{0,0}\right)+\ldots \text { etc },
\end{aligned}
$$

where $\mu_{x} \delta_{x} f_{r, s}=\frac{1}{2}\left(\delta_{x} f_{r+1,8}+\delta_{x} f_{r-1, s}\right)$ and so for $\mu_{y} \delta_{y}$.

To apply this formula, take trapezoidal sums of columns and rows and form difference tables with these as before. For differences as far as the third, corner terms being neglected, it gave for $\int_{-4}^{.8} \int_{1 \cdot 3}^{1 \cdot 8} e^{x^{4} y} d x d y$ the value correct to five places, $\cdot 36591$.

Central difference formulae of numerical integration are rapidly convergent, differences of even order not appearing. In the corresponding interpolation formulae no differences of odd order appear, so that, if a function is to be tabulated for the double purpose of interpolation and numerical integration, as useful a table as any is one giving the values of the function and of all the central differences as far as required.

\$7. Formulae in Differential Coefficients.

The double Euler-Maclaurin formula is

$$
\begin{array}{r}
\frac{1}{u v} \int_{a}^{a+m u} \int_{b}^{b+n v} f(x, y) d x d y=S_{u}^{m} S_{v}^{n}-\frac{u}{12} \cdot \frac{\partial}{\partial x}\left[S_{u}^{0} S_{v}^{n}\{f(a+m u, b)\right. \\
-f(a, b)\}]-\frac{v}{12} \cdot \frac{\partial}{\partial y}\left[S_{u}^{m} S_{v}^{0}\{f(a, b+n v)-f(a, b)\}\right]
\end{array}
$$




$$
\begin{aligned}
& +\frac{u^{3}}{720} \cdot \frac{\partial^{3}}{\partial x^{3}}\left[S_{u}^{0} S_{v}^{n}\{f(a+m u, b)-f(a, b)\}\right] \\
& +\frac{v^{3}}{720} \cdot \frac{\partial^{3}}{\partial y^{3}}\left[S_{u}^{m} S_{v}^{0}\{f(a, b+n v)-f(a, b)\}\right]
\end{aligned}
$$

- etc. ... $+\frac{u v}{144} \cdot \frac{\partial^{2}}{\partial x \cdot \partial y}[f(a+m u, b+n v)-f(a, b+n v)-f(a+m u, b)$

$$
+f(a, b)]+\ldots
$$

The correction terms here, as in previous formulae, are taken in columns and rows on the boundary, summed trapezoidally, together with small and generally negligible corner terms.

The formula gave for $\int_{0}^{1} \int_{0}^{1}\left(1+x^{2}+y^{2}\right)^{-\frac{3}{2}} d x d y$ the value 523604, partial coefficients as far as the third being taken, and 121 values of the integrand being tabled.

$$
\int_{0}^{1} \int_{0}^{1} e^{x^{2} y} d x d y \text { and } \int_{0}^{\cdot 8} \int_{0}^{1 \cdot 2} e^{x^{2} y} d x d y
$$

were also calculated for 121 and 117 points respectively, results being obtained correct to five places, 1.20702 and $1 \cdot 10469$.

\section{\$8. General Conclusions.}

Comparisons of the rapidity and accuracy of calculations performed by means of all the foregoing formulae of double numerical integration seem to show that the best method is to apply one or other of the finite difference formulae to trapezoidal sums of the rows and columns concerned. For moderately close approximations, however, a double Simpson or a Gauss formula may serve.

In conclusion we wish to thank Professor E. T. Whittaker, at whose suggestion this work was undertaken, for valuable advice given from time to time during its preparation. 